\title{
MERCATI E COMPETITIVITÀ IN TRANSIZIONE
}

\author{
Daniele Dalli
}

La rivista, insieme alla sua comunità di riferimento $\mathrm{e}-$ in definitiva $-\mathrm{a}$ tutta l'università, sta vivendo una fase di transizione che da un lato preoccupa e mette in crisi modelli consolidati e dall'altra sembra assai promettente per lo sviluppo di modelli nuovi e più efficaci. Per modelli intendo le modalità con cui gli accademici e le relative istituzioni si relazionano con l'ambiente di riferimento e rispondono alle sue sollecitazioni o cercano di anticiparle o condizionarle.

Non è questa la sede per discorsi di tipo politico o istituzionale su cui la rivista si è comunque soffermata in passato. In questo Editoriale vorrei sottoporre all'attenzione della comunità che si riconosce nella Società Italiana di Marketing (SIMktg) e nella Rivista alcune questioni pratiche relativamente al contesto attuale e agli scenari futuri.

Nel corso del convegno di Modena c'è stata l'occasione per introdurre alcuni dei nodi cruciali che riguardano la Rivista e in particolare la questione della visibilità nei database citazionali. Dopo due anni di lavoro alla guida di $\mathrm{M} \& \mathrm{C}$ posso dire che la situazione è decisamente migliorata, in egual misura per a) l'impegno del precedente Direttore (Annalisa Tunisini), b) il lavoro svolto sulla Rivista in questo periodo e c) l'azione di proselitismo svolta in collaborazione con la SIMktg. I soci e gli abbonamenti sono aumentati, il numero di articoli ricevuti è stabile e migliora la qualità media dei manoscritti, il lavoro dei revisori è nettamente migliorato, dando il senso di un circuito virtuoso che si sta piano piano consolidando dopo notevoli sforzi iniziali.

Parallelamente si è intensificata l'azione istituzionale per la promozione e supporto della rivista sia in seno all' Aidea che di concerto con la Società Italiana di Management (SIMA) con cui la Rivista collabora per varie iniziative.

Il risultato di cui sono più orgoglioso è la crescita dell'indice $\mathrm{H}$ che in pochi mesi (Febbraio-Settembre 2014) è salito di 2 punti. Non riporto il dato assoluto per una questione di affidabilità dei dati e perché è soggetto a oscillazioni significative. E anche per scaramanzia, visto che vorrei che

Mercati e Competitività n. 4, 2014 
continuasse a crescere con questo ritmo. Evidentemente il posizionamento della Rivista comincia a recepire i risultati degli sforzi effettuati e la richiesta ai Soci della SIMktg e più in generale a tutti coloro che si identificano in $\mathrm{M} \& \mathrm{C}$ di citare la rivista nei propri lavori di ricerca produce gli effetti sperati.

In questo periodo si è cercato anche un maggiore coinvolgimento dei colleghi attraverso la richiesta di Call for Papers presso i responsabili delle Sezioni Tematiche della SIMktg, ma non solo. In questo senso, i network tra ricercatori che si vogliono proporre per arricchire la Rivista sono ben accolti e questo costituisce un canale importante per fare proselitismo, soprattutto nei confronti dei colleghi stranieri, sia come autori che come lettori e quindi come fonte di citazioni.

La dimensione internazionale dello sviluppo della conoscenza costituisce un aspetto imprescindibile del lavoro degli studiosi di management e di marketing in particolare, per una serie di ragioni che non sto qui a prendere in rassegna tanto sono numerose e variegate, oltre che arcinote ai componenti della nostra comunità. È per questo motivo che la SIMktg ha deliberato di orientare in tal senso la Rivista in modo ancora più radicale, prevedendo che nel 2015 si raggiunga un 100\% di materiali scritti in lingua inglese. Questa scelta è assai rischiosa e prevede alcuni costi, sia per la Rivista che per gli autori, ma se riusciamo in questa operazione potremmo in breve tempo beneficiare di ottimi risultati. E ciò riguarda la Rivista e la SIMktg, ma soprattutto la comunità accademica e in particolare gli autori. $\mathrm{M} \& \mathrm{C}$ non farà concorrenza al Journal of Marketing, ma potrebbe in breve costituire un'alternativa alle molte riviste internazionali di livello medio attualmente disponibili, ma dietro alle quali non esiste un progetto editoriale specifico, né un profilo culturale identificabile.

Lo sviluppo della rivista, peraltro, non si riduce alla traduzione dei testi e alla "chiamata alle armi" dei colleghi nella guerra delle citazioni. Servono linee guida in ambito editoriale che facciano crescere i contenuti e diano alla Rivista una personalità sempre più spiccata e riconoscibile. Anzitutto merita osservare che una spinta notevole al miglioramento dei contenuti viene dall'interazione tra autori e revisori, mediato dalla redazione: posso affermare che dal punto di vista metodologico la qualità dei materiali in entrata è assai migliorata proprio grazie al lavoro di educazione che $\mathrm{i}$ revisori e per tramite loro la Rivista hanno svolto.

In secondo luogo, per quanto riguarda i contenuti, ritengo che sia necessario agire in due direzioni. Primo, per ciò che riguarda la progettazione editoriale dei saggi per $M \& C$ proporrei agli autori due direttrici:

- Una logica top down in cui si segue un filone di ricerca che si evolve a livello internazionale e al quale si cerca di contribuire senza un particolare riferimento alla nostra realtà territoriale e culturale. Questo è un 
percorso piuttosto consolidato all'estero e molti colleghi italiani, soprattutto giovani, lo seguono con grande efficacia. Ci sono alcuni aspetti critici dell'attuale dibattito che si sviluppa sulle riviste di marketing e in particolare sulla capacità della nostra disciplina di influire efficacemente sugli sviluppi teorici di ordine superiore. Chi è interessato a seguire questa logica dovrebbe leggere con attenzione il recente contributo di Clark et al. (2014) sulla capacità della ricerca di marketing di influire sulle altre business disciplines.

- Una logica bottom up, ovvero cercando di inserire in un dibattito internazionale il proprio contributo che può avere una sua specificità rispetto al contesto di riferimento. Se vogliamo presentare un profilo culturale distintivo ai lettori stranieri, dobbiamo anche valorizzare quegli aspetti della nostra realtà territoriale e culturale che possono interessare il dibattito internazionale. Industrie, prodotti, brand, tendenze che si sviluppano nel nostro paese possono e devono essere valorizzate e collocate in un dibattito più ampio. Spesso è difficile trovare spazio per questi contributi sulle riviste straniere, mentre su M\&C questi articoli saranno assai apprezzati, nel rispetto dei consueti criteri di adeguatezza teorica e di metodo e se adeguatamente collocati nella letteratura di riferimento.

Secondo, non è pensabile che la Rivista sia rivolta solo ed esclusivamente a una platea accademica e per questo sono necessari aggiustamenti e integrazioni. In un mercato editoriale internazionale, i lettori interessati a $\mathrm{M} \& \mathrm{C}$ possono essere anche professionisti e manager, forse anche più di quanto non accada oggi in Italia. Più in generale, progettare i propri articoli con un occhio al dibattito teorico e uno alla rilevanza operativa sembra necessario dato che, come rilevato dal recente lavoro di Roberts et al. (2014a) le due cose sono strettamente collegate. Ancorché debole, esiste una correlazione diretta tra impatto accademico e impatto professionale e cosa ancor più significativa - quanto più importante è un' area decisionale nei processi di marketing aziendali, tanto più importante è il ruolo in essa giocato dai contributi teorici della letteratura di marketing. Sembra pertanto che il trade off tra rigore e rilevanza spesso citato in chiave quasi esclusivamente politica o ideologica nei nostri discorsi accademici possa essere declinato in una chiave più moderna e costruttiva $\mathrm{e}$ - diciamolo - più intelligente. Da un lato la ricerca rigorosa fine a se stessa non serve a nessuno e nemmeno agli stessi accademici di marketing (vedi il paper di Clark et al. citato sopra) e dall'altro non esiste una ricerca rilevante che non sia anche rigorosa. Per ulteriori elementi si vedano anche i commenti all'articolo originale riportati in bibliografia.

Su questo versante e sempre nel rispetto dei criteri di adeguatezza teorica e metodologica dei contributi, segnalo due opportunità che contribuireb- 
bero non poco allo sviluppo del profilo e alla differenziazione di M\&C nel panorama internazionale e cioè:

- I nostri colleghi francesi hanno percorso la strada della traduzione in inglese prima di noi e il nuovo direttore della RAM (Recherche et Applications en Marketing) ha impresso un'ulteriore sterzata in questa direzione grazie al contributo di colleghi stranieri, i cui contributi (brevi approfondimenti di 4 o 5 pagine) sono contenuti in una sezione ad hoc intitolata Expert opinions. Possiamo procedere anche noi in questa direzione e coinvolgere non solo studiosi stranieri, ma anche professionisti e manager, italiani e non, in collaborazione con accademici italiani o da soli, che vogliano condividere con i nostri lettori i loro punti di vista su aspetti correnti o in corso di evoluzione delle proprie attività di ricerca o professionali. Coloro che hanno contatti che puntino in questa direzione sono invitati a contattarmi per proposte e chiarimenti del caso.

- Esiste una forte domanda, sia sul versante della ricerca che della formazione e della professione, di casi di studio. Di recente anche in settori vicini (organizzazione e management) si assiste a un recupero del metodo dei casi e delle metodiche qualitative che consentono un accesso ai problemi teorici e pratici piuttosto efficace oltre a contribuire all'identificazione di sviluppi teorici da testare ulteriormente con altre metodologie. Alcuni giovani accademici sono al lavoro su questo versante e sarebbero interessati a pubblicare i propri casi di studio. Anche in questo caso sarà necessaria una sezione ad hoc per presentare a una platea internazionale interessanti casi di marketing italiani, sia che siano circoscritti al contesto nazionale o che descrivano un percorso di progressiva apertura verso i mercati internazionali. Questa pratica editoriale trova la sua origine in Economia \& Management che molti di noi considerano un'ottima rivista per la capacità di declinare teoria e pratica professionale e dalla quale utilmente si può imparare. Anche in questo caso sono a disposizione di chi fosse interessato per proposte e chiarimenti.

Naturalmente i materiali di cui sopra devono essere in inglese e passare attraverso un processo di revisione che sarà allineato a quello tradizionale, ma con parametri diversi in merito alla valutazione da parte dei revisori.

\section{Riferimenti bibliografici}

Clark T., Key T., Hodis M., Rajaratnam D. (2014). The intellectual ecology of mainstream marketing research: an inquiry into the place of marketing in the family of business disciplines. Journal of the Academy of Marketing Science, 42 (3): 223-241. 
Lehmann D.R. (2014). Commentary on "From academic research to marketing practice: Exploring the marketing science value chain" (John H. Roberts, Ujwal Kayande, Stefan Stremersch), International Journal of Research in Marketing, 31 (2): 141-141.

Roberts J.H., Kayande U., Stremersch S. (2014a). From academic research to marketing practice: Exploring the marketing science value chain. International Journal of Research in Marketing, 31 (2): 127-140.

Robert, J.H., Kayand, U., Stremersch, S. (2014b). From academic research to marketing practice: Some further thoughts. International Journal of Research in Marketing, 31 (2): 144-146. 